\title{
Pancreatic dysfunction and its association with fat malabsorption in HIV infected children
} A Carroccio, M Fontana, M I Spagnuolo, G Zuin, G Montalto, R B Canani, F Verghi,
D Di Martino, K Bastoni, F Buffardi, A Guarino malabsorption..$^{2-5}$ Steatorrhoea is a prominent feature of intestinal dysfunction. Faecal fat concentration is frequently notably increased in HIV infected children, resembling that found in coeliac disease. ${ }^{6}$ The pathophysiology of intestinal dysfunction is largely unknown. Intestinal damage (partial villous atrophy and crypt hypertrophy) is frequently found in HIV infected children. ${ }^{7}$ However there is no definite proof that steatorrhoea is related to intestinal damage. ${ }^{8}$

Pancreatic disease is also a frequent finding in AIDS. Hyperamylasaemia has been reported in approximately half of HIV infected patients, without specific symptoms of pancreatic disease, ${ }^{9}$ and autopsy examinations of AIDS patients often reveal pancreatic abnormalities. ${ }^{10}$ Most of the information available on pancreatic disease derives from adult patients ${ }^{9-11}$; there is little information on children. Miller et al published a retrospective study on 53 children followed for six years, in nine of whom $(17 \%)$ pancreatitis was detected. ${ }^{12}$ However, no data have been published on pancreatic digestive function.

A prospective case controlled study was performed to investigate exocrine pancreatic function in children with symptomatic HIV infection. The hypothesis that pancreatic involvement could be associated with steatorrhoea was also tested. atic enzymes were not associated with symptoms. Twelve children had steatorrhoea and four had increased $\alpha_{1}$ antitrypsin. Steatorrhoea was significantly associated with reduced faecal pancreatic enzymes. There was a significant negative correlation between elastase 1 concentration and steatocrit. Children with pathological faecal elastase 1 or chymotrypsin values did not differ from the other HIV infected children with respect to nutritional and immunological status, stage of HIV disease, presence of opportunistic infections, or drug administration. Conclusions-Abnormal pancreatic function tests are a frequent feature of paediatric HIV infection; this condition is associated with steatorrhoea, which probably contributes to the disease. (Gut 1998;43:558-563)

Keywords: HIV; children; pancreas; steatorrhea; malnutrition; intestine

Intestinal malabsorption is frequent in both adult and paediatric patients with symptomatic HIV infection. ${ }^{12}$ In the latter, it consists of faecal nutrient loss and intestinal iron

\section{Patients and methods}

PATIENTS

Forty seven children (24 males, 23 females; median age 7.3 years, range 1-16) were enrolled between November 1996 and April 1997 at two tertiary care centres for paediatric HIV infection. The following information was obtained for each child: HIV class according to the Center for Disease Control (CDC) criteria $^{13}$; CD4 lymphocyte count; patient's weight expressed as weight $\mathrm{z}$ score; presence of opportunistic infections; drug treatment, including zidovudine, dideoxyinosine, cotrimoxazole, and pentamidine; presence of gastrointestinal symptoms, including diarrhoea defined as three or more unformed or liquid stools per day) and abdominal pain (defined as a pain episode limiting normal daily activity or causing wakening during sleep with a frequency of at least one bout per week during the previous three months). Weight $\mathrm{z}$ score was calculated as follows, according to our previous study ${ }^{14}:$ z score $=$ (observed weight - mean weight for sex and age)/standard deviation for sex and age; Italian regional standards were used as reference values. 
Forty five age and sex matched children were enrolled as controls. They had been admitted to the Di Cristina Hospital to undergo surgery for inguinal or umbilical hernia or for cryptorchidism, and had no history of intestinal disease. Informed consent was obtained from the parents or the legal guardians of the children.

PANCREATIC FUNCTION TESTS

Pancreatic function was investigated by two non-invasive tests: the quantitative determination of elastase 1 (EL-1) and chymotrypsin $(\mathrm{CMT})$ activities in faecal specimens. Stools were collected for 24 hours from children receiving a standard diet (with carbohydrates $50-60 \%$ of total energy, proteins $12-15 \%$, and fats approximately $30 \%$ ); stools were subsequently homogenised, weighed, and stored at $-20^{\circ} \mathrm{C}$. An aliquot was used to determine faecal enzymes; this was performed by the Division of Internal Medicine Laboratory of the University of Palermo. Another aliquot was used to determine lipid and faecal $\alpha_{1}$ antitrypsin concentration; this was performed by the Department of Pediatrics Laboratory of the University of Naples.

EL-1 activity was determined by a commercially available enzyme linked immunosorbent assay (ELISA), which uses two monoclonal antibodies against two distinct specific epitopes of human pancreatic elastase (Schebo-tech, Wettenberg, Germany). Results were expressed as $\mu \mathrm{g} / \mathrm{g}$ stool or as $\mu \mathrm{g} / 24 \mathrm{~h}$. The lower normal limit of EL-1 was $200 \mu \mathrm{g} / \mathrm{g}$ stool or 10000 $\mu \mathrm{g} / 24 \mathrm{~h}^{15} \mathrm{CMT}$ activity was determined at $37^{\circ} \mathrm{C}$ by a photometric assay (Monotest Chymotrypsin, Boehringer, Mannheim, Germany). Results were expressed as U/g stool or as units $/ 24 \mathrm{~h}$. The lower normal limit was 7.5 $\mathrm{U} / \mathrm{g}$ or $375 \mathrm{U} / 24 \mathrm{~h}$. The cut off limits were derived from data obtained in the same laboratory in 300 age matched healthy controls. ${ }^{16}$ Both EL-1 and CMT activities were determined on specimens kept frozen for up to 30 days. Previous data showed that cold storage did not modify results, the interassay coefficient for variation being $9.8 \%$ for CMT and $8.3 \%$ for EL-1. Finally, after an overnight fast, a sample of venous blood was taken to determine serum total amylase and pancreatic amylase activities, using commercially available colorimetric methods (Amyl and P-Amyl, respectively; Boehringer-Mannheim, Mannheim, Germany). According to data obtained in the same laboratory from a large group of age matched healthy subjects, the higher normal limits were $160 \mathrm{U} / 1$ for total amylase and $83 \mathrm{U} / 1$ for pancreatic amylase.

INTESTINAL FUNCTION TESTS

Intestinal function was investigated by determining faecal nutrient loss, as previously described. ${ }^{2}$ Faecal fat excretion was quantitatively estimated by the steatocrit method, as previously reported. ${ }^{17}$ Results were expressed as amount of increase over normal age related values and a result greater than the mean (2 SD) of age matched controls was considered abnormal. ${ }^{17}$ It has been previously shown that the mean interday coefficient of variation can be as low as $2 \%$ and does not exceed $20 \%{ }^{17}$; therefore the 24 hour steatocrit can be regarded as having a narrow variability range. Intestinal protein loss was assessed by the quantitative determination of $\alpha_{1}$ antitrypsin in stools by a radial immunodiffusion assay, as previously described..$^{18}$ A result greater than 0.9 $\mathrm{mg} / \mathrm{g}$ stool was considered abnormal. ${ }^{18}$ D-xylose oral load was performed in 15 children as part of an investigation of intestinal malabsorption and $20 \mathrm{mg} / \mathrm{dl}$ was the cut off limit. $^{19}$

\section{STATISTICAL ANALYSIS}

The Mann-Whitney U test was used to evaluate the difference between pancreatic enzyme activities in HIV infected children and controls. The same test was used to compare the weight z score, CDC class, and CD4 count in patients with low faecal pancreatic enzymes and in patients with normal enzymes. Spearman's correlation coefficient was applied to determine the significance of the correlation between steatocrit and faecal EL-1 and CMT activities, respectively. The $\chi^{2}$ test evaluated the significance of the different prevalences of fat malabsorption, diarrhoea, abdominal pain, opportunistic infections, or drug administration in children with normal or abnormal faecal pancreatic enzymes.

\section{Results}

The 47 children enrolled were classified as follows, according to their HIV infection state: 16 C3, five $\mathrm{C} 2$, eight $\mathrm{B} 3$, two $\mathrm{B} 2$, four B1, three A3, four A2, two A1, one N2, and two N1. Fourteen $(30 \%)$ had abnormally low faecal EL-1 and/or CMT activities. Of these 14 children, seven had isolated EL-1 deficiency, three isolated CMT deficiency, and four deficiency in both pancreatic enzymes.

CMT was therefore abnormally low in seven children $(15 \%)$ whereas none of the controls had abnormal CMT activities. The mean CMT activity was significantly lower in HIV infected children than in controls $(95 \%$ confidence interval 9.0 to $19.6 ; \mathrm{z}=4.65$; $\mathrm{p}<0.0001$; Mann-Whitney U test) (fig 1). The same results were obtained by expressing CMT activity as output/24 h: mean (SD) CMT output was 1809 (1510) in HIV infected children and 2620 (1305) $\mathrm{U} / 24 \mathrm{~h}$ in controls $(\mathrm{z}=3.17$; $\mathrm{p}<0.0001$; Mann-Whitney U test).

In $11 \mathrm{HIV}$ children (23\%) faecal EL-1 was below the lower limit; none of the controls had abnormal EL-1 activities. The mean EL-1 activity was significantly lower than that of controls (95\% confidence interval 108.8 to 253.2; $\mathrm{z}=4.19 ; \mathrm{p}<0.0001$, Mann-Whitney U test) (fig 2). Similar results were obtained when EL-1 was expressed as total faecal output/24 h: the same 11 children had abnormally low EL-1 output and the mean (SD) value was 32160 (18 650) versus $50320 \quad(20$ 520) ( $\mathrm{z}=4.28$; $\mathrm{p}<0.0001$; Mann-Whitney U test).

Serum amylase values were elevated in 12 children and in these patients the values were 1.2-4-fold higher than the normal limit; only one of these 12 patients had low faecal pancreatic enzymes. There was therefore no correla- 


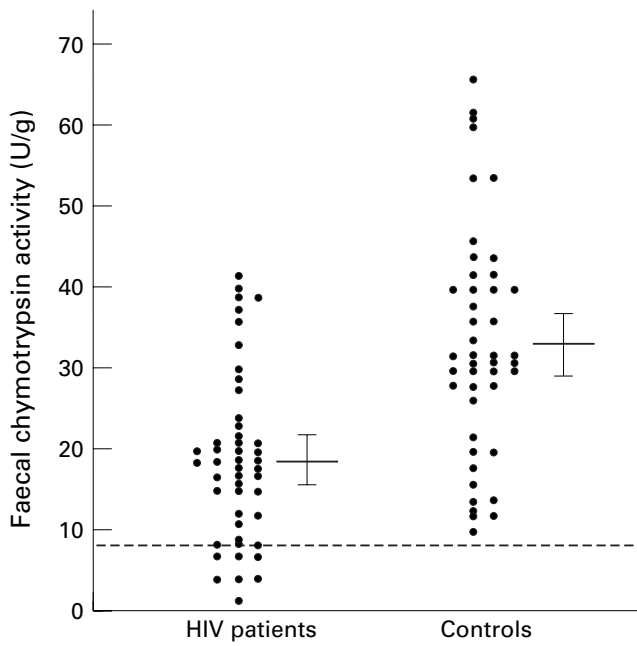

Figure 1 Individual and mean values of faecal chymotrypsin activity. Dotted line indicates lower normal limit. Bars indicate mean values and $95 \%$ confidence intervals.

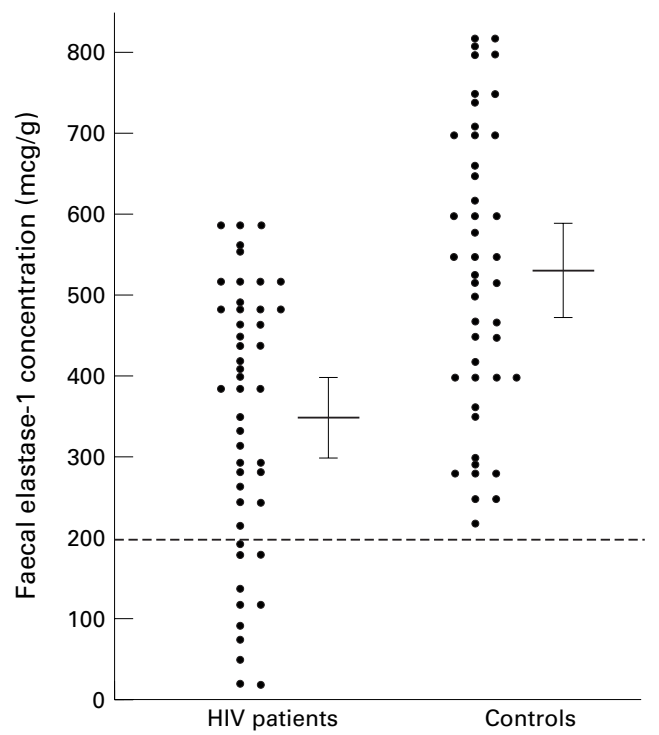

Figure 2 Individual and mean values of faecal elastase 1. Dotted line indicates lower normal limit. Bars indicate mean values and $95 \%$ confidence intervals.

tion between serum amylase activity and pancreatic function tests. Furthermore, the serum pancreatic isoamylase measurements revealed that the increased enzyme activity was of salivary origin in all but two cases.

Twelve of the $47 \mathrm{HIV}$ patients (26\%) had fat malabsorption as shown by the elevated steatocrit values; in controls this test was consistently within normal limits. The mean value (SD) of steatocrit was an 11 (7)-fold increase over age reference values in the HIV infected children. Figure 3 shows the relation between fat malabsorption and pancreatic function tests in the $47 \mathrm{HIV}$ patients studied. Fat malabsorption was detected in 8/14 children with low faecal pancreatic enzyme activities $(57 \%)$ and in $4 / 33$ children with normal faecal pancreatic enzymes (12\%). The prevalence of steatorrhoea was significantly $(p<0.01)$ increased in patients with abnormal pancreatic function tests. Furthermore, there was a significant association between steator-

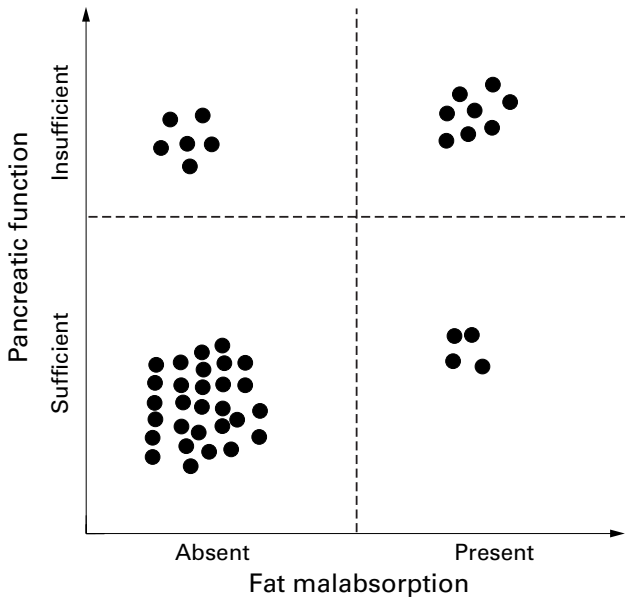

Figure 3 Relation between faecal fat content and pancreatic deficiency (reduced faecal CMT and/or EL-1 activities) in HIV infected patients.

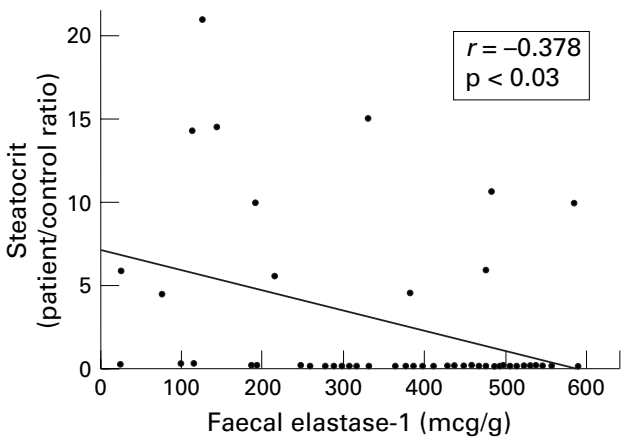

Figure 4 Regression analysis of faecal elastase 1 and steatocrit values in HIV infected patients.

rhoea and low EL-1 activity, but not CMT activity. Figure 4 shows the quantitative relation between EL-1 and steatorrhoea: there was a significant negative correlation between steatocrit and faecal EL-1 values in the HIV infected children $(r=-0.34 ; \mathrm{p}<0.03$; intercept 378.9 (SE 26.3); slope -12.5 (SE 5.48)).

Four children had protein loss, judged by the increased $\alpha_{1}$ antitrypsin concentration in their stools. One also had steatorrhoea, but none showed reduced faecal pancreatic enzyme concentration. Following the D-xylose oral load, $5 / 15$ patients $(30 \%)$ had a low blood concentration. Three of these five had low pancreatic enzymes in stools.

At the time of the study eight children had diarrhoea and nine abdominal pain. Diarrhoea was not significantly different between children with low pancreatic enzymes and those with normal pancreatic enzymes (4/14 versus $4 / 33$, $\mathrm{p}>0.05)$. Abdominal pain was not different in children with low or normal faecal pancreatic enzymes (3/14 versus $6 / 44, p>0.05$ ).

Finally, the nature of pancreatic involvement was further investigated by assessing its relation with HIV infection status, as well as the role of selected opportunistic agents, which has been shown in pancreatic disease in HIV infected adults. ${ }^{20}$ The possibility that some of the drugs that are frequently used in HIV infected patients may induce a reduction in faecal pancreatic enzymes was also investigated. Table 1 shows the individual features, 
Table 1 Patient characteristics

\begin{tabular}{|c|c|c|c|c|}
\hline Patient no & Weight $z$ score & CD4 count & Infection & Drugs \\
\hline \multicolumn{5}{|c|}{ Abnormal pancreatic function tests } \\
\hline 1 & -0.42 & 275 & CMV & ZDV, DDI, pentamidine \\
\hline 2 & +0.38 & 161 & CMV, candida & ZDV, DDI, cotrimoxazole prednisolone \\
\hline 3 & +1.61 & 544 & & DDI, cotrimoxazole \\
\hline 4 & -1.87 & 15 & Herpes & DDI, pentamidine \\
\hline 5 & -3.25 & 11 & Herpes, candida & DDI \\
\hline 6 & -0.79 & 1796 & & ZDV, DDI, pentamidine \\
\hline 7 & -3.3 & 10 & Pneumocystis carinii & ZDV, DDI \\
\hline 8 & -1.2 & 1197 & & ZDV \\
\hline 9 & -0.66 & 1796 & & ZDV \\
\hline 10 & -1.41 & 400 & $\mathrm{CMV}$ & ZDV, DDI, prednisolone \\
\hline 11 & +2.13 & 390 & & \\
\hline 12 & +0.08 & 2442 & & ZDV, DDI, prednisolone \\
\hline 13 & +0.59 & 475 & CMV, herpes & ZDV, DDI \\
\hline 14 & -0.66 & 1152 & & \\
\hline Median & -0.66 & 437.5 & & \\
\hline Range & -3.3 to +2.1 & $10-2442$ & & \\
\hline \multicolumn{5}{|c|}{ Normal pancreatic function tests (data of other 33 patients) } \\
\hline Median & -0.47 & 360.0 & & \\
\hline Range & -3.8 to +2.5 & $6-3258$ & & \\
\hline
\end{tabular}

Opportunistic infections: cytomegalovirus (CMV), 8 cases; Epstein Barr virus, 3 cases; herpes virus, 2 cases; toxoplasma, 2 cases; candida, 2 cases; Pneumocystis carinii, 6 cases.

Drug administration: zidovudine (ZDV), 27 cases; dideoxyinosine (DDI), 16 cases; pentamidine, 9 cases; cotrimoxazole, 9 cases; lamivudine, 5 cases; prednisolone, 2 cases; captopril, 1 case.

Table 2 Faecal pancreatic enzyme activity subdivided according to HIV clinical categories

\begin{tabular}{lcccc}
\hline & $\begin{array}{l}\text { Mild or no signs/symptoms } \\
(\text { CDC class } A)\end{array}$ & $\begin{array}{l}\text { Moderate signs/symptoms } \\
\text { (CDC class B) }\end{array}$ & $\begin{array}{l}\text { Severe signs/symptoms } \\
\text { (CDC class C) }\end{array}$ & Total \\
\hline Enzyme activity & 10 & 10 & 13 & 33 \\
$\quad$ Normal & 2 & 4 & 8 & 14 \\
$\quad$ Low & 12 & 14 & 21 & 47 \\
$\quad$ Total & & & \\
\hline CDC classification. ${ }^{13}$ & & & & \\
p $>0.05 \chi^{2}$ test for trend. & &
\end{tabular}

including weight $\mathrm{z}$ score, CD4 cell count, opportunistic infections, and drug treatment of the 14 children with low pancreatic enzyme stool concentrations. No difference was observed between these 14 children and all the others studied for any of the parameters considered. In particular, with respect to infections, cytomegalovirus infection prevalence was not different in children with or without pancreatic enzyme abnormalities (4/14 versus 8/33, p>0.05). None of the children with low pancreatic enzymes had Epstein Barr virus infection. With regard to drug administration, the number of children receiving pentamidine isothionate or dideoxyinosine was similar in those with or without pancreatic enzyme abnormalities (3/14 children with low pancreatic enzymes were on pentamidine versus $9 / 33$ children with normal pancreatic enzymes; the corresponding figures for dideoxyinosine were $10 / 14$ and 16/33) indicating that pancreatic dysfunction is not related to selected drugs.

Table 2 shows HIV infection status of the children according to the CDC scheme for clinical features ${ }^{13}$ and its relation with pancreatic enzymes. Pancreatic dysfunction prevalence was higher in children with clinically severe HIV infection than in those with mild or moderate disease, although the difference was not statistically significant.

\section{Discussion}

HIV infection results in a multisystemic disorder involving the gastrointestinal tract and malabsorption syndrome is often observed. ${ }^{1-3}$ In HIV infected children intestinal dysfunction is associated with faecal nutrient loss. It is of note that intestinal dysfunction is not consistently associated with diarrhoea ${ }^{34}$ although it certainly contributes to weight loss and ultimately to the terminal cachexia in HIV infected patients. However, the cause-effect relation between intestinal epithelial damage, malabsorption, and nutrient loss is far from being established..$^{1-48}$

Patients with AIDS have an increased risk of developing pancreatic disease. ${ }^{11}$ Autopsy studies have shown severe pancreatic lesions in a large number of these patients, ${ }^{91120}$ including 61/73 HIV infected children without clinical evidence of pancreatic disorders. ${ }^{21}$ However, pancreatic lesions are rarely associated with symptoms and therefore pancreatic disease is frequently not recognised. ${ }^{9} 12021$ One infant with HIV infection and severe fat malabsorption was found to have severe pancreatic fibrosis and normal jejunum at autopsy, ${ }^{22}$ suggesting that fat malabsorption may be a direct consequence of pancreatic disease in paediatric AIDS.

We carried out the first prospective study on pancreatic function in HIV infected children and tested the hypothesis that fat malabsorption could be related to pancreatic dysfunction. To do so, the concentrations of two specific pancreatic enzymes were determined in the stools, together with intestinal function tests performed on faecal specimens. This allowed us to investigate pancreatic function and its relation with intestinal function in a noninvasive manner in children with serious clinical conditions. We must emphasise that a single determination of faecal pancreatic en- 
zyme concentration cannot be considered a definitive evaluation of exocrine pancreatic function, but it should be noted that faecal EL-1 is a novel, highly sensitive, and specific pancreatic function test ${ }^{15}$ which has a very high correlation with the duodenal output of all pancreatic enzymes after the secretin-caerulein test $^{23}$ and it has been used to monitor pancreatic function in children with cystic fibrosis. ${ }^{24} 25$

Our data showed that one third of our HIV infected children had low faecal enzyme activities, indicating impaired pancreatic function. EL-1 activities were more frequently altered than CMT and it is likely that this reflects a higher sensitivity of EL-1 than of CMT in patients with mild pancreatic disease. ${ }^{23}$ Results expressed as daily output were identical to those expressed as concentration, thus adding to the consistency of the data.

The hypothesis that the abnormal pancreatic function test results could be associated with fat malabsorption was then tested. Previously, it had been suggested that HIV infection can cause pancreatic insufficiency and malabsorption, ${ }^{9}{ }^{10} 11$ but only one report specifically studied this relation. ${ }^{25}$ In that study, $12 / 25$ adults with HIV infection had fat malabsorption and only three had exocrine pancreatic insufficiency, as judged by the tyrosylPABA test ${ }^{25}$; it was concluded that mild exocrine pancreatic deficiency syndrome may occur in HIV infection, but fat malabsorption is more commonly associated with a small intestinal disorder. ${ }^{26}$ However, at present, it is not known whether there is really no correlation between pancreatic dysfunction and fat loss in HIV infected adults; it may not have been detected because of a limited sample size in that study or because of a limited sensitivity of the tyrosyl-PABA test in identifying pancreatic dysfunction. ${ }^{26}$

Our findings show that a relation exists between fat loss and abnormal pancreatic function test results in HIV infected children and suggest that pancreatic dysfunction may contribute to fat loss in stools. In fact, approximately two thirds of the children with steatorrhoea also had reduced pancreatic enzyme concentration in stools. Furthermore, we found a statistically significant correlation between steatocrit and EL-1 values $(r=-0.34)$; as impairment of pancreatic function is not the only hypothetical cause of fat malabsorption -intestinal mucosal damage is well known in HIV patients-it is not surprising that the correlation we found was weak and suggested that a reduction in pancreatic enzyme activities is only one of a number of factors determining fat malabsorption.

Finally, although our evaluation of intestinal absorption was incomplete (the xylose test was performed in 15 patients only), it could be significant that $3 / 5 \mathrm{HIV}$ infected children with abnormal xylose absorption also had low faecal pancreatic enzymes. This resembles coeliac disease, a condition in which pancreatic impairment secondary to intestinal mucosal damage has been documented. ${ }^{1626}$
With regard to serum amylase activities, we found an increase in 12/47 HIV infected children, but none of them had clinical evidence of pancreatitis; furthermore, the amylase isoenzyme study showed that only $2 / 12$ patients had an elevated pancreatic isoamylase, whereas in all other cases the increase was of salivary origin. Consequently, as expected, there was no correlation between serum amylase activities and pancreatic digestive capacity in the study patients, and we can suggest that serum amylase determination is not useful in the identification of HIV patients with pancreatic insufficiency.

With regard to the clinical consequence of reduced faecal pancreatic enzymes, although the prevalence of either diarrhoea or abdominal pain was higher in children with low pancreatic enzymes, this was not statistically significant. More data are needed to ascertain whether or not this condition is associated with gastrointestinal symptoms.

Further studies are also needed to clarify the pathogenesis of pancreatic dysfunction. In fact, the hypothesis that low pancreatic enzymes might be related to severe HIV infection was not proved in this study. The lack of a significant association is possibly due to the relatively limited sample size, as the number of children with low enzymes progressively increased from those with mild to moderate and to severe HIV infection. However the CD4 cell count did not support this hypothesis. With regard to cytomegalovirus (CMV) infections, involved in pancreatic diseases in adult HIV infection patients, ${ }^{20}$ we found that CMV infection frequency was higher in subjects with low faecal pancreatic enzymes, but the difference was not statistically significant. Therefore the role of CMV was not proved and longitudinal studies are needed to determine whether CMV is able to induce pancreatic diseases in children with HIV infection. The role of drugs is far less clear; it has been suggested that pentamidine may induce pancreatitis in children with a low CD 4 cell count, ${ }^{12}$ but we found no evidence for this or for other drugs in HIV infected children.

In conclusion, this study shows that a reduction in faecal pancreatic enzymes is a common feature in paediatric HIV infection and it is associated with fat loss; results suggest that therapeutic intervention could be effective in children with exocrine pancreatic insufficiency. Enzyme replacement therapy could be an effective treatment for HIV infected subjects with pancreatic dysfunction; the effectiveness of such treatment is currently under investigation.

This was a collaborative study of the Italian Society of Pediatric Gastroenterology and Hepatology. The work was supported by grants from the Italian Health Ministry: AIDS research project 1996, program 9404-32, and program 9401-19.

\footnotetext{
1 Sharpstone D, Gazzard B. Gastrointestinal manifestations of HIV infection. Lancet 1996;348:379-83.

2 Guarino A, Albano F, Tarallo L, et al. Intestinal malabsorption of HIV-infected children: relationship to diarrhoea, failure to thrive, enteric micro-organism and immune failure to thrive, enteric micro-or
impairment. AIDS 1993;7:1435-40

3 Yolken RH, Hart W, Oung I, et al. Gastro-intestinal dysfunction and disaccharide intolerance in children infected with human immunodeficiency virus. $f$ Pediatr 1991;118:359-63.
} 
4 Miller TL, Orav J, Martin SR, et al. Malnutrition and carbohydrate malabsorption in children with vertically transmithydrate malabsorption in children with vertically transmit-
ted human immunodeficiency virus 1 infection. Gastroented human immunodeficiency

terology 1991;100:1296-302.
5 Castaldo A, Tarallo L, Palomba E, et al. Iron deficiency and intestinal malabsorption in HIV disease. F Pediatr Gastroenterol Nutr 1996;22:359-63.

6 Guarino A, Tarallo L, Guandalini S, et al. Impaired intestinal function in symptomatic HIV infection. $\mathcal{f}$ Pediatr Gastroenterol Nutr 1991;12:453-8.

7 Chang T, Pelton SI, Winter HS. Enteric infections in HIVinfected children. In: Blaser MJ, Smith PD, Ravdin JI, et al,
eds. Infections of the gastrointestinal tract. New York: Raven Press, 1995:499-510

8 Keating J, Bjarnason I, Somasundaram S, et al. Intestinal absorptive capacity, intestinal permeability and jejunal histology in HIV and their relation to diarrhoea. Gut 1995;37: tology in

9 Brivet F, Coffin B, Bedossa P, et al. Pancreatic lesions in AIDS. Lancet 1987; ii: $570-1$

10 Zazzo JF, Pichon F, Reigner B. HIV and the pancreas. Lancet 1987;ii: 1212-13.

11 Cappell MS, Hassan T. Pancreatic disease in AIDS-a review. F Clin Gastroenterol 1993;17:254-63.

12 Miller TL, Winter HS, Luginbuhil LM, et al. Pancreatitis in pediatric human immunodeficiency virus infection. F Pediatr 1992;120:223-7.

13 Center for Disease Control and Prevention. Revised classification system for human immunodeficiency virus infection in children less than 13 years of age. MMWR 1994;43:117.

14 Carroccio A, Iacono G, Montalto G, et al. Pancreatic enzyme therapy in childhood celiac disease. Dig Dis Sci 1995;40:2555-60.

15 Stein J, Jung M, Sziegoleit A, et al. Immunoreactive elastase I: clinical evaluation of a new non-invasive test of pancreatic function. Clin Chem 1996;42:222-6.
16 Carroccio A, Iacono G, Lerro P, et al. Role of pancreatic impairment in growth recovery during gluten-free diet in 44 .

17 Guarino A, Tarallo L, Greco L, et al. Reference values of the steatocrit and its modifications in diarrheal diseases. F Pediatr Gastroenterol Nutr 1992;14:268-74.

18 Catassi C, Cardinali E, D'Angelo G, et al. Reliability of random alpha1-antitrypsin determination in non-dried stools. 7 Pediatr 1986;3:500-2.

19 Craig RM, Atkinson AJ Jr. D-xylose testing: a review. Gastroenterology 1988;95:223-31.

20 Wilcox CM, Forsmark CE, Grendell GH, et al. Cytomegalovirus-associated acute pancreatic disease in patients with acquired immunodeficiency syndrome: report of two patients. Gastroenterology 1990;99:263-7.

21 Bricaire F, Marche C, Zoubi D, et al. HIV and the pancreas. Lancet 1988;i:65-6.

22 Kahn E, Anderson VM, Greco MA, et al. Pancreatic disorders in pediatric acquired immune deficiency syndrome. Hum Pathol 1995;26:765-70.

23 Torre D, Montanari M, Fiori PG, et al. HIV and the pancreas. Lancet 1987;ii:1212.

24 Loser Chr, Mollgard A, Folsch UR. Fecal elastase 1: a novel, highly sensitive, and specific tubeless pancreatic function test. Gut 1996;39:580-6.

25 Terbrack HG, Gurtler KH, Klor HU, et al. Human pancreatic elastase 1 concentration in faeces of healthy children and children with cystic fibrosis [abstract]. Gut 1995; 37(suppl 2):A253

26 Kapembwa MS, Fleming SC, Griffin GE, et al. Fat absorption and exocrine pancreatic function in human immunodeficiency virus infection. $Q \mathcal{F} \mathrm{Med}$ 1990;74:49-56.

27 Carroccio A, Iacono G, Montalto G, et al. Exocrine pancreatic function in children with celiac disease before and after a gluten-free diet. Gut 1991;32:796-9. 